Supporting Information for

\title{
Temperature-induced Transition from Indirect to Direct Adsorption of Polycyclic Aromatic Hydrocarbons on Quartz: A Combined Theoretical and Experimental Study
}

Tu Lan, ${ }^{\dagger, \star \S}$ Jing Liu, ${ }^{\dagger}$ Hongbo Zeng, ${ }^{*}, \dagger$ and Tian Tang,,$*$

${ }^{\dagger}$ Department of Chemical and Materials Engineering, University of Alberta, Edmonton, Alberta T6G 1H9, Canada

${ }^{\ddagger}$ Department of Mechanical Engineering, University of Alberta, Edmonton, Alberta T6G 1H9, Canada

${ }^{\S}$ Key Laboratory of Radiation Physics and Technology of the Ministry of Education, Institute of Nuclear Science and Technology, Sichuan University, Chengdu 610064, P. R. China

*Corresponding authors:

Phone: +1-780-492-1044. E-mail: hongbo.zeng@ualberta.ca (H.Z.).

Phone: +1-780-492-5467. E-mail: tian.tang@ualberta.ca (T.T.). 


\section{Section S1. Initial Configuration}

Figure S1 shows the initial configuration in system H323, consisting of 24 VO-79 molecules with their PACs parallel to one another, forming a $4 \times 2 \times 3$ array. Two quartz surfaces were placed on the left and right sides of the box, with the $\mathrm{Si}-\mathrm{OH}$ groups facing and interacting with heptane. Other systems shared the same initial configuration.

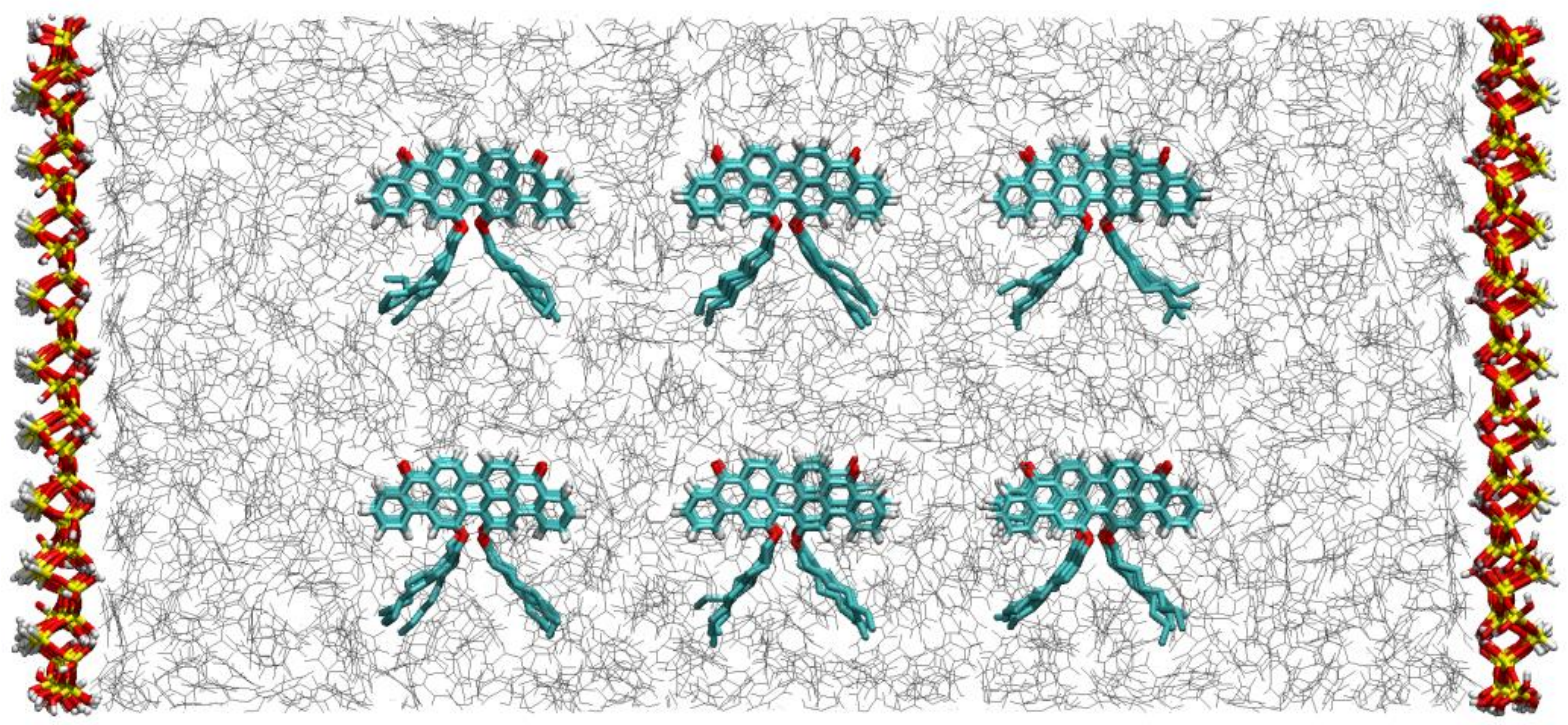

Figure S1. Initial configuration in system H323. 


\section{Section S2. RMSD}

In Figure S2, the root mean square deviations (RMSD) of VO-79 molecules are plotted as functions of time, which clearly demonstrate the attainment of equilibrium for all systems in the end. In heptane (Figure S2a), for systems H323, there is little change in RMSD after $60 \mathrm{~ns}$. With the increase of temperature, it takes less time $(<40 \mathrm{~ns})$ to reach equilibrium for the other four systems. Similar trend is observed in toluene (Figure S2b). These results are not unexpected, because rising temperature can significantly increase the diffusion rate of VO-79 molecules, ${ }^{\mathrm{S} 1}$ leading to more contact and faster adsorption on quartz surface. Once adsorbed, their mobility will be reduced quickly.
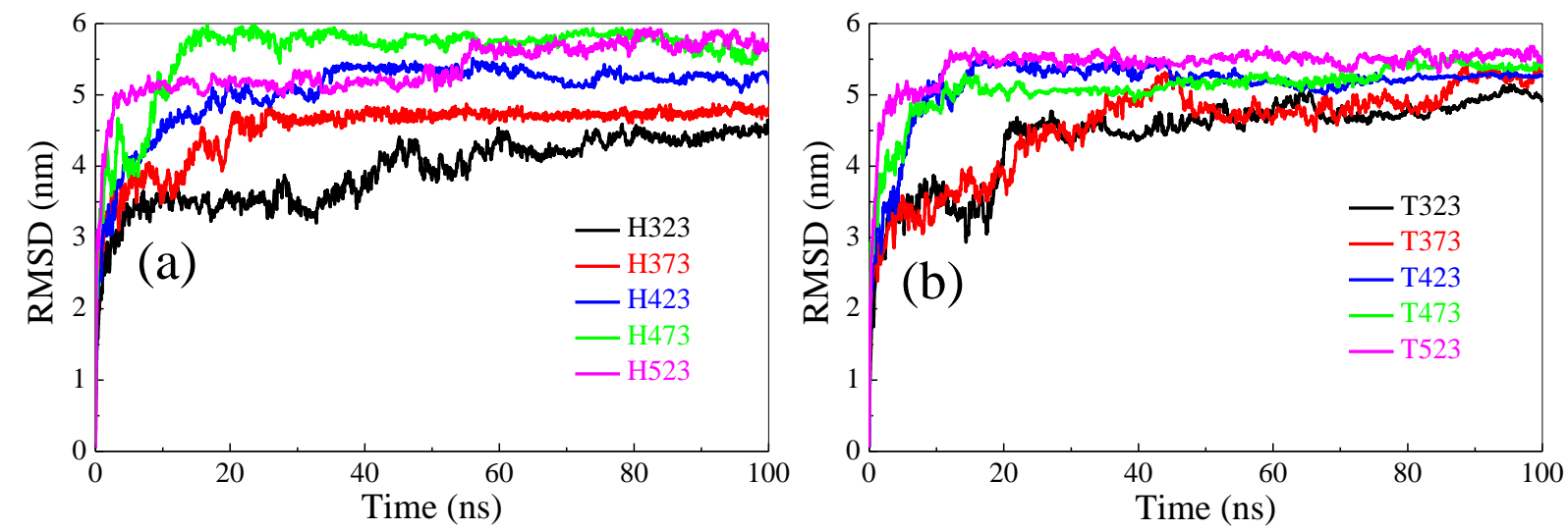

Figure S2. Time evolution of root mean square deviation (RMSD) of VO-79 molecules in (a) heptane and (b) toluene at different temperatures. 


\section{Section S3. Minimum Distance}

Figure S3 shows the minimum distance between the PAC of each VO-79 and quartz surface $\left(d_{1}\right)$ as a function of time in systems H323 and T323 at $323 \mathrm{~K}$. For most VO-79, $d_{1}$ decreases with time until reaching an equilibrium value of $\sim 0.25 \mathrm{~nm}$.
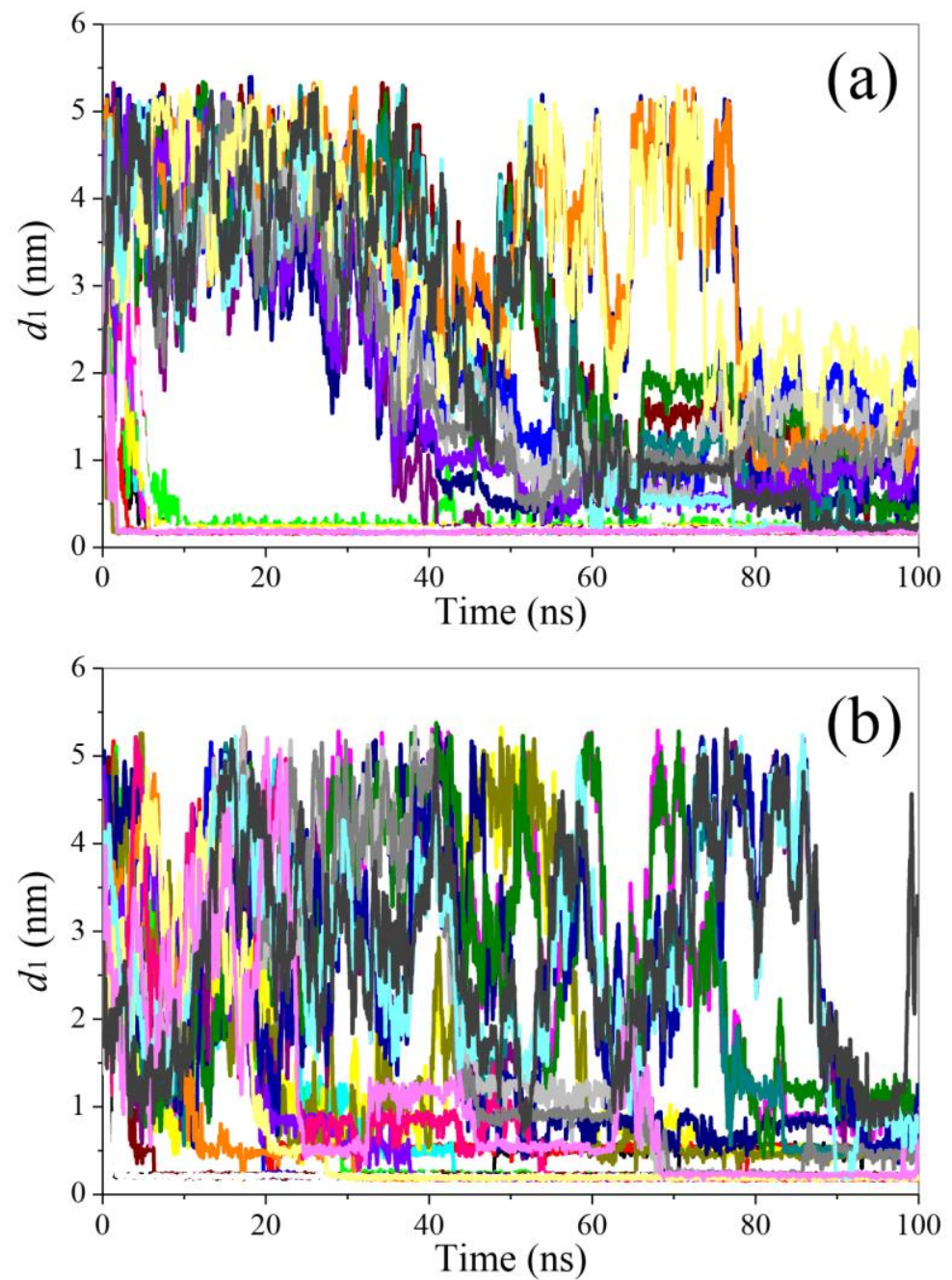

Figure S3. Minimum distance $\left(d_{1}\right)$ between the PAC of each VO-79 and quartz surface in (a) heptane and (b) toluene at $323 \mathrm{~K}$. 
Figure S4 shows the number of VO-79 molecules adsorbed on quartz surface from the solution at different simulation time. Solid columns correspond to the number of molecules with direct adsorption while shaded columns correspond to the number of molecules with indirect adsorption.
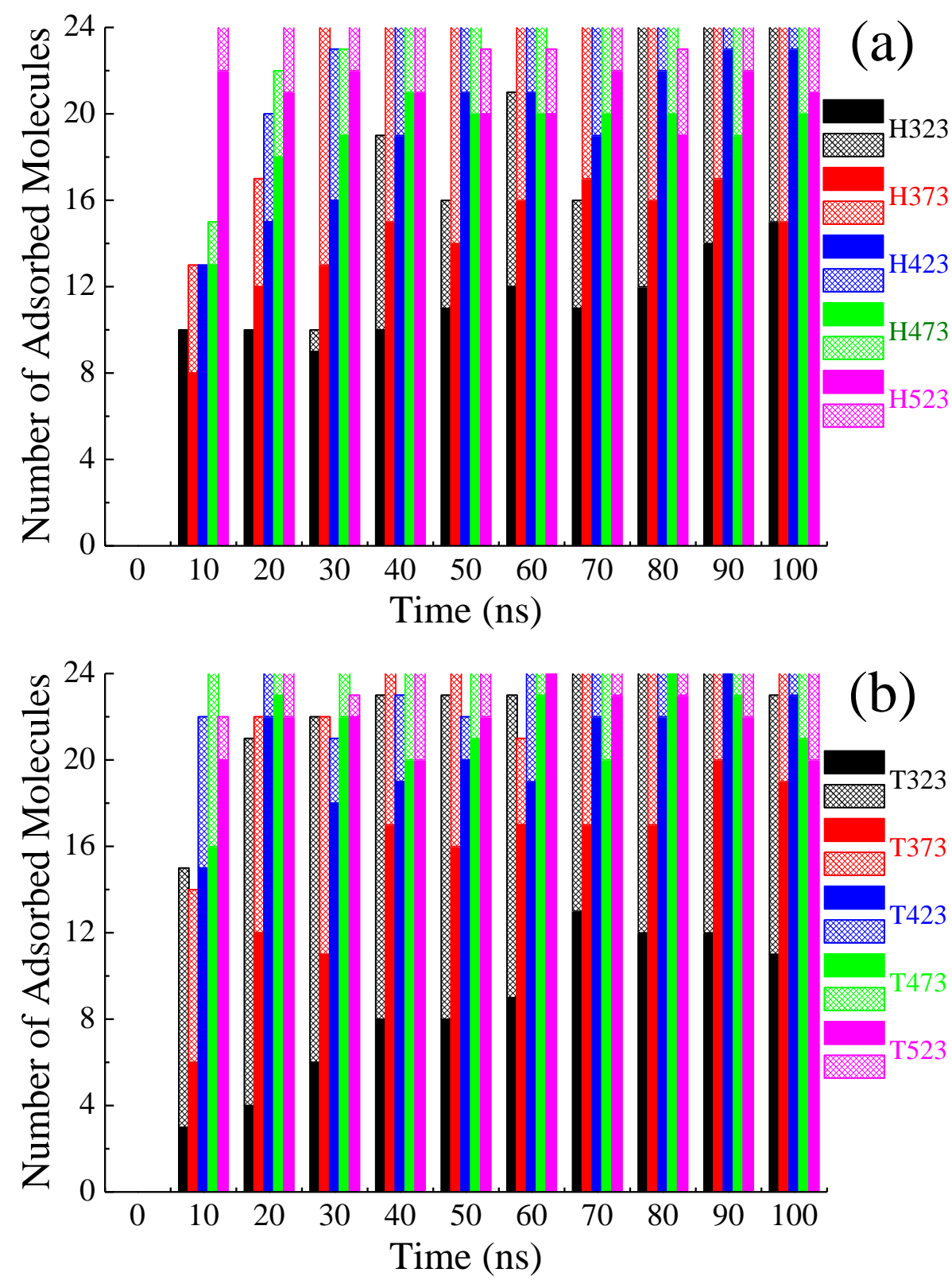

Figure S4. Number of VO-79 molecules adsorbed on quartz surface from (a) heptane and (b) toluene at different simulation time.

In heptane at $323 \mathrm{~K}(\mathrm{H} 323)$, the fastest direct adsorption appeared in the first $10 \mathrm{~ns}$ as the number of directly adsorbed VO-79 increased from 0 to 10 . The maximum rate can thereby be 
estimated to be $10 /(10 \mathrm{~ns})=1.0 \mathrm{~ns}^{-1}$. The direct adsorption reached a maximum of 15 at 100 $\mathrm{ns}$, and the average rate was calculated to be $15 /(100 \mathrm{~ns})=0.15 \mathrm{~ns}^{-1}$. For other systems in heptane, the fastest direct adsorption also occurred in the first $10 \mathrm{~ns}$ regardless of temperature, and the maximum rates for $\mathrm{H} 373, \mathrm{H} 423, \mathrm{H} 473$, and $\mathrm{H} 523$ are $0.8,1.3,1.3$, and $2.2 \mathrm{~ns}^{-1}$, respectively. However, the time taken to reach a maximum of direct adsorption was different for the 4 systems, and the average rates were calculated to be $0.24,0.26,0.53$, and $2.2 \mathrm{~ns}^{-1}$, respectively, for system $\mathrm{H} 373, \mathrm{H} 423, \mathrm{H} 473$, and $\mathrm{H} 523$. 


\section{Section S5. Calculation of the Effective Thickness of Adsorbed VO-79 Layers}

Figure S5 shows the mass density of VO-79 in heptane and toluene at different temperature in the direction $(z)$ perpendicular to the quartz surfaces. The two quartz surfaces are located at $z=0$ and $z=12.4 \mathrm{~nm}$, respectively. For each system, the density distributions of VO-79 show two peaks near the two surfaces but become zero at a certain distance away from the surfaces. The distance near each quartz surface over which the VO-79 density is non-zero is used as the effective thickness of the adsorbed VO-79 layer on this surface. For example, the VO-79 density of system H323 in Figure S5a is non-zero from $0.37 \mathrm{~nm}$ to $4.57 \mathrm{~nm}$ near the left surface and from $9.52 \mathrm{~nm}$ to $11.99 \mathrm{~nm}$ near the right surface. The effective thickness is therefore $(4.57-0.37) \mathrm{nm}=4.20 \mathrm{~nm}$ and $(11.99-9.52) \mathrm{nm}=2.47 \mathrm{~nm}$ respectively for the two adsorbed layers. The data are collected in Table S1 for the thicknesses on the left and right surfaces and their sum (total thickness).
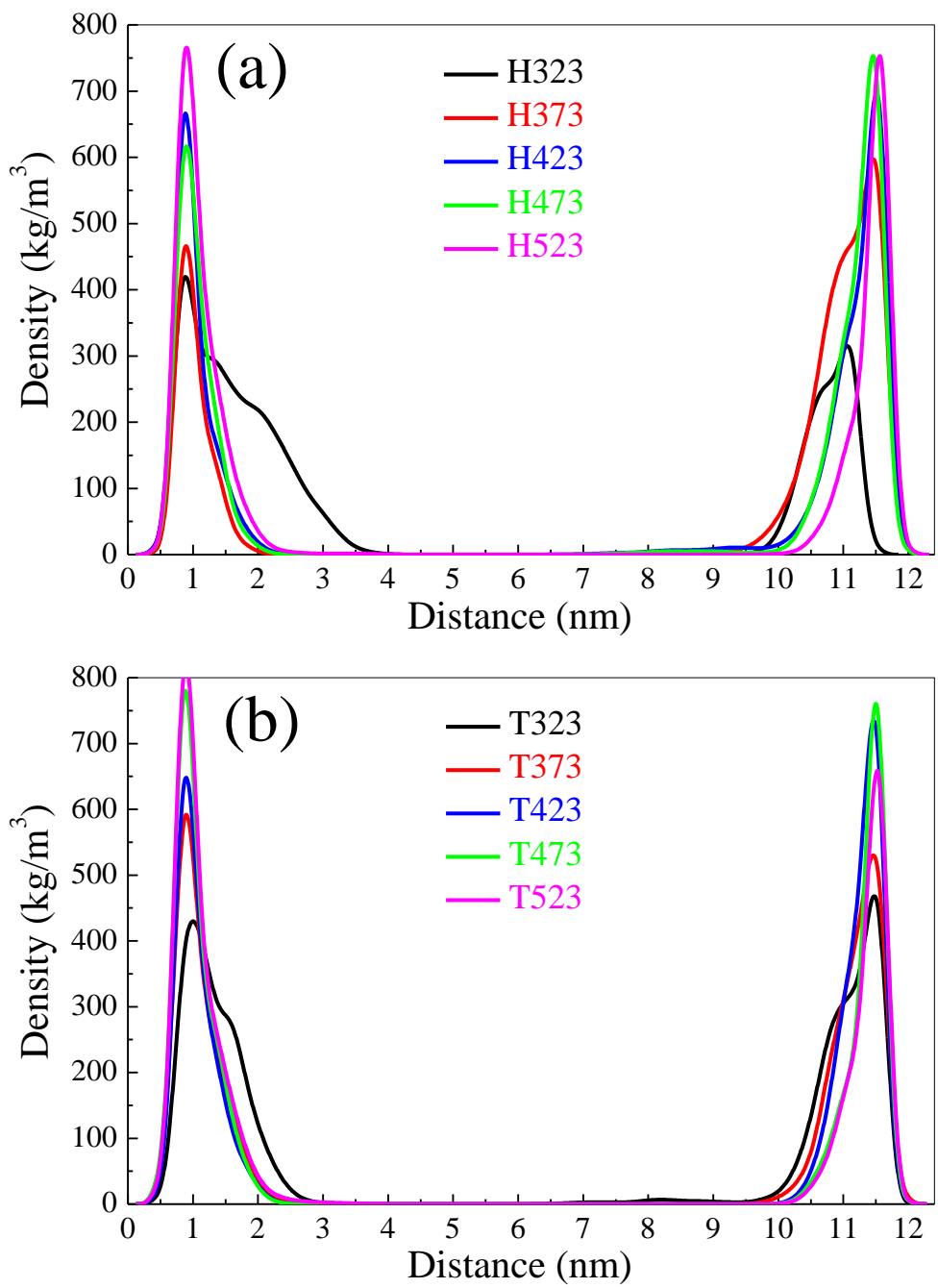

Figure S5. Mass density of VO-79 in (a) heptane and (b) toluene at different temperatures in the direction $(z)$ perpendicular to the quartz surfaces, averaged over the last $5 \mathrm{~ns}$ of the simulations. 
Table S1. Effective thickness of the adsorbed VO-79 layers (nm) on quartz surfaces

\begin{tabular}{cccc}
\hline systems & left layer & right layer & total thickness \\
\hline H323 & 4.20 & 2.47 & 6.67 \\
H373 & 1.98 & 3.97 & 5.95 \\
H423 & 2.23 & 2.23 & 4.46 \\
H473 & 2.23 & 2.22 & 4.45 \\
H523 & 2.24 & 2.24 & 4.48 \\
T323 & 2.98 & 2.73 & 5.71 \\
T373 & 2.48 & 2.73 & 5.21 \\
T423 & 2.23 & 2.23 & 4.46 \\
T473 & 2.23 & 2.23 & 4.46 \\
T523 & 2.23 & 2.23 & 4.46 \\
\hline
\end{tabular}




\section{Section S6. Free Energy of Solvation}

The free energies of solvation $\left(G_{\text {solv }}\right)$ of VO-79 molecules were calculated as functions of time based on the solvent accessible surface area (SASA), ${ }^{\mathrm{S} 2}$ and the results are plotted in Figure S6. As VO-79 begin to adsorb on the quartz surfaces, $G_{\text {solv }}$ reduces quickly and reaches equilibrium in the end. The change in $G_{\text {solv }}\left(\Delta G_{\text {solv }}\right)$ was calculated as the difference between the average in the first $0.1 \mathrm{~ns}$ and the average in the last $5 \mathrm{~ns} . \Delta G_{\text {solv }}$ in heptane and toluene are respectively in the range of -442.79 to $-239.17 \mathrm{~kJ} / \mathrm{mol}$ and -365.35 to $-228.75 \mathrm{~kJ} / \mathrm{mol}$, which are comparable to the energy contributed by electrostatics (Figure 8 in the main text).
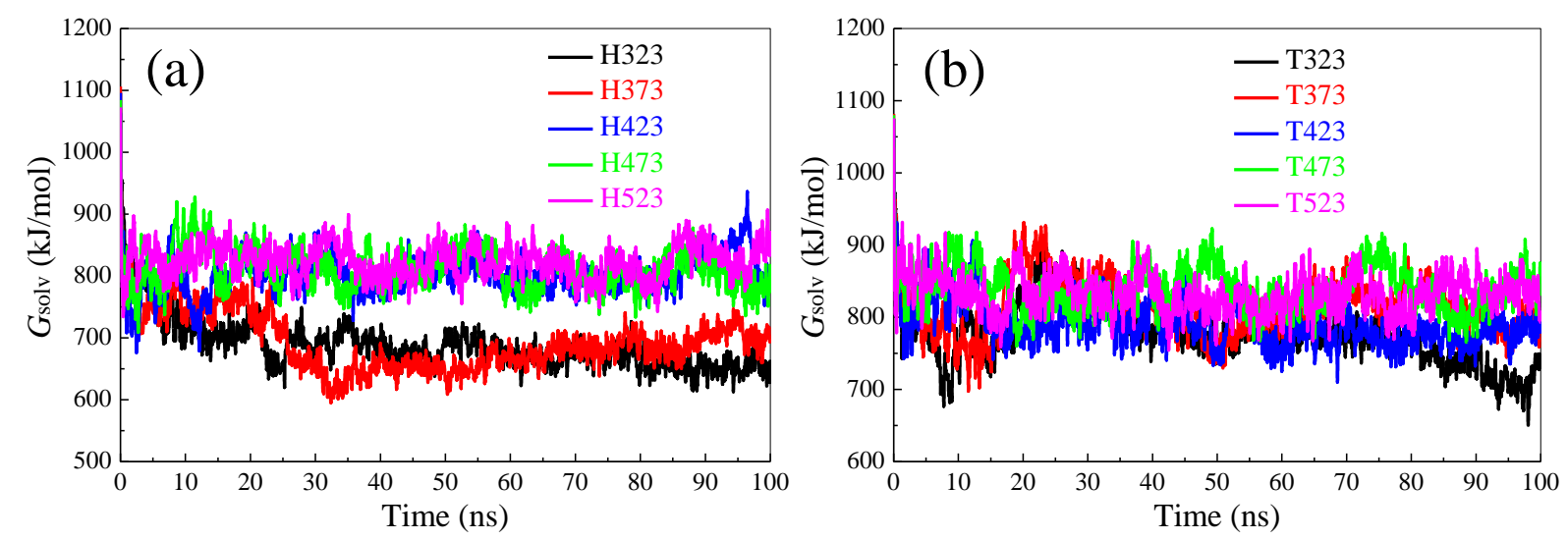

Figure S6. Free energy of solvation $\left(G_{\text {solv }}\right)$ of VO-79 molecules in (a) heptane and (b) toluene as functions of time. 


\section{References}

(S1) Adams, J. J. Asphaltene adsorption, a literature review. Energy Fuels 2014, 28, 2831-2856.

(S2) Eisenhaber, F.; Lijnzaad, P.; Argos, P.; Sander, C.; Scharf, M. The double cubic lattice method: Efficient approaches to numerical integration of surface area and volume and to dot surface contouring of molecular assemblies. J. Comput. Chem. 1995, 16, 273-284. 